\title{
The Research of Impact of Quantum Entanglement and Decoherence on Determinative Remote Preparation
}

\author{
Zhaohua Wang ${ }^{\text {a }}$ Liqing Ren, Hanying Wang \\ College of Energy Engineering, Yulin University, Shaanxi 719000, China \\ awangzhaohua841102@163.com
}

Keywords: quantum entanglement, determinative, remote preparation, impact

\begin{abstract}
In this paper, two problems of quantum entanglement and decoherence are analyzed. Firstly, remote implementation scheme is analyzed, and situation of bit consumption is grasped after the scheme is finalized. Then, the entanglement elimination and coherence phenomenon are analyzed from the analysis and remote preparation process. And through the influence of this phenomenon on the system, it is known that in the process of polarization, the choice of the longitude of the target quantum in the Bloch sphere gradually does not relate to the fidelity of the system, but only relate to the size of the decoherence.
\end{abstract}

\section{Introduction}

In the field of quantum information subject, two main practical methods are quantum teleportation and remote preparation [1]. Based on the local operation, the sending end sends the quantum to the receiving end Bob through Alice. The practical purpose of remote preparation is to transfer quantum states [2]. This article will analyze this remote preparation scheme to obtain deterministic conditions and analyze the impact of target bits on system fidelity [3].

\section{Determinative remote preparations scheme}

Table 1 The type of remote preparation scheme

\begin{tabular}{ll}
\hline Preparation type & Classical quantum states \\
Determinative preparation
\end{tabular}

\subsection{The way of classical quantum states}

In the present study, the target qubits are assumed, ie, $\varphi \geq \cos \frac{\theta}{2}$ and $\varphi \geq \sin \frac{\theta}{2}$, which is represented by the Bloch sphere. Thereinto, the values range is from 1 to $\pi$, range of $\varphi$ is from 0 to $2 \pi$.In this formula, $\theta$ represents the latitude value of the quantum state in the sphere, and $\varphi$ represents the longitude value of the quantum state in the sphere[4]. Based on these two parameters, any quantum state in the sphere can be confirmed. Bloch ball is as shown in Figure 1[5].

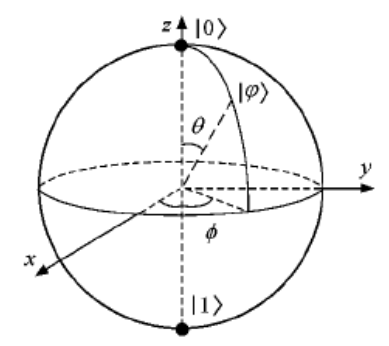

Fig. 1 Schematic diagram of Bloch ball 
When preparing classical quantum state, from the perspective of the sending end, the longitude and latitude of the quantum state in the sphere belong to the known state. In order to transmit $\varphi$ to the receiving end successfully, the transmission can be achieved only when both transmit in the same quantum channel. For example, select one among the Bell bases and expand it to obtain another form. The details are as shown in Table 2 [1].

Table 2 The opening and closing style of the selected formula

\begin{tabular}{cc}
\hline Time & Formula \\
\hline Before the expansion & $\varphi \geq \frac{1}{\sqrt{2}}(|01\rangle|10\rangle)_{A B}$ \\
After the expansion & $\varphi=\frac{1}{\sqrt{2}}\left(|\varphi\rangle_{A}|\varphi\rangle_{B}-|\varphi\rangle_{B}\right)$ \\
\hline
\end{tabular}

In the sending end, after measuring the particles in the hand by projection, if the obtained structure is larger than the numerical value $\varphi_{A}$,it means that the quantum state size obtained at the receiving end is also that $A$ is greater than $B$; comparing with the value, if the structure is small, then it indicates that the size of the quantum state obtained in the receiving end is also that $\mathrm{A}$ is less than $\mathrm{B}$; From the quantum state, it can be seen that there is hardly any transform leading the result in which value $\varphi$ is greater than $\mathrm{A}$. So if situations above occur on the sending side, it means that the remote preparation is unsuccessful and the failure rate is $50 \%$. However, some operations on quantum state have been proposed in the relevant literature and can be used to achieve the above operation. For example, in the spherical representation, when the position of the quantum state is at the pole or the equator, it can be realized that the value of $\varphi$ is larger than A in the corresponding sending end. But it completes this remote preparation at the expense of 1 quantum bit on the sending end.

\subsection{Determinative preparation}

For different schemes, there are some differences in the probability of success in the implementation of remote preparation. At this stage, after the effective improvement of the preparation scheme, the success rate of preparation has been significantly improved. In the related literature, this issue in this area has been studied, and developed a way that makes POVM measurements on a particle A of sending end. The main measurement is shown in Figure 2.

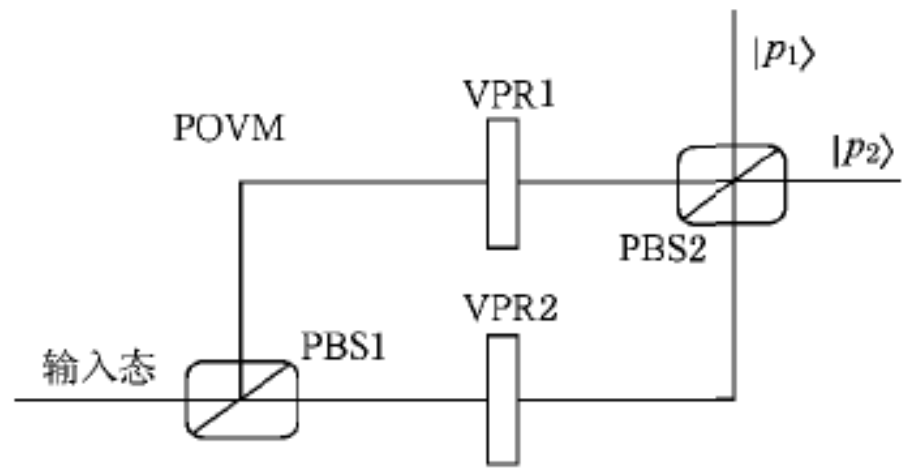

Fig.2 POVM measurement method

As can be seen from the figure above, the main function of the VPR is to shift the polarization state by effectively adjusting the polarization rotation. The main PBS serves as a polarizing beam splitter. After two functions of VPR, the quantum state has changed in varying degrees, as shown in Table 3. After measuring the quantum through the method in Fig. 1, the entanglement pairs have also changed to some extent, as shown in Table 4. 
Table 3 Changes in quantum state

\begin{tabular}{cc}
\hline & Changes \\
\hline VPR1 & $H>\cos \frac{\theta}{2}$ \\
VPR2 & $V>\cos \frac{\theta}{2}$ \\
\hline & Table 4 Changes in entanglement pairs \\
\hline VPR1 & Changes \\
\hline VPR2 & $H=\frac{1}{\sqrt{2}} \cos \frac{\theta}{2}$ \\
& $V=\frac{1}{\sqrt{2}} \cos \frac{\theta}{2}$ \\
\hline
\end{tabular}

\section{Analysis of the influence of entanglement and decoherence}

Deterred preparation is carried out in the above manner, which occurs mainly when entangled pairs exist in the systematic quantum channels. However, from the objective reality, the state of entanglement is very unstable. Under the influence of the environment, it is very easy to produce entanglement and coherence. The decoherence mentioned in this paper is mainly divided into two types, as shown in Table 5.

Table 5 Type of entanglement and decoherence

\begin{tabular}{cc}
\hline Type & $\begin{array}{c}\text { Polarized decoherence } \\
\text { Phase decoherence }\end{array}$ \\
\hline
\end{tabular}

\subsection{Polarized decoherence}

In the process of the implementation of this way, we should ensure that $\mathrm{P}$ and $\varphi$ are always in the same state. In the appearance of this ball, once the location of the target quantum state reaches the extreme point, it will make fidelity decreased. And with its continuous movement to the equator, fidelity is gradually improved. When it reaches the equator, the fidelity is also the maximum. Then it gradually away from the equator, fidelity begin to show a downward trend, which is as shown in Fig.3[2].

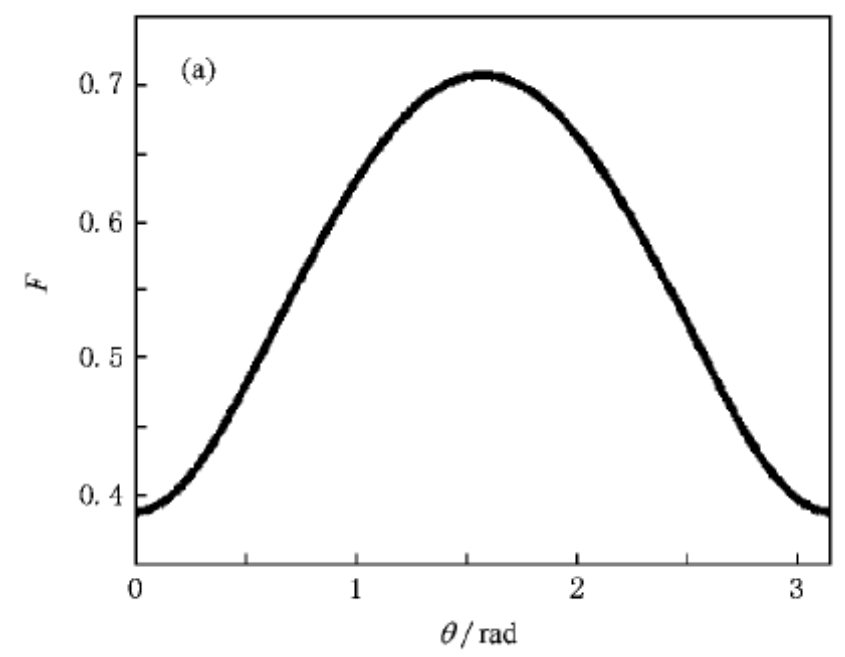

Fig.3 Changing trend chart of fidelity in polarized decoherence 


\subsection{Phase decoherence}

From the above analysis and deduction, it can be concluded that under this type of decoherence, there is no link between the system fidelity and the target quantum, and therefore the change in longitude cannot be shown in the sphere at the same time, there is no connection between the size of the decoherence, which can be drawn from Figure 2. The main reason for the above phenomenon is that the structure of POVM possesses symmetry. They can offset each other in the function of phase decoherence. So its size and fidelity will gradually be related, and then make an impact.

\section{Conclusions}

In summary, in the subject of quantum information, two ways of the main application are quantum teleportation and remote preparation. This article mainly analyzes two kinds of outstanding problems about quantum entanglement and decoherence. First, the remote implementation scheme is analyzed. After analyzing the phenomenon of entanglement and decoherence in the whole process of analysis and remote preparation, we conclude that in the process of polarization, the longitude selection of the target quantum in Bloch ball is not linked with the fidelity of the system gradually, and it is only linked with the size of decoherence.

\section{Acknowledgements}

This work was supported by Shaanxi science and technology research and development plan project: 2016GY-196; Shaanxi science and technology research and development plan project: 2014K05-11.

\section{References}

[1] Pourkarimi M R, Rahnama M, Rooholamini H. Decoherence Effect on Quantum Correlation and Entanglement in a Two-qubit Spin Chain[J]. International Journal of Theoretical Physics, 2015, 54(4):1085-1097.

[2] Liang H Q, Liu J M, Feng S S, et al. Effects of noises on joint remote state preparation via a GHZ-class channel[J]. Quantum Information Processing, 2015, 14(10):3857-3877.

[3] Tsai C W, Lin J. Fault-tolerant Remote Quantum Entanglement Establishment for Secure Quantum Communications[J]. International Journal of Theoretical Physics, 2016, 55(7):3200-3206.

[4] Caputa P, Simón J, Štikonas A, et al. Quantum entanglement of localized excited states at finite temperature[J]. Journal of High Energy Physics, 2015, 2015(1):1-28.

[5] Jean Michel Raimond, Paolo Facchi, Bruno Peaudecerf, et al. Quantum Zeno dynamics of a field in a cavity[J]. Physical Review A, 2012, 86(3):9591-9598. 